\title{
Evaluation of the Cementation Index as a Predictor of Failure in Coonrad-Morrey Total Elbow Arthroplasty
}

\author{
Manish Kiran, ${ }^{1}$ Arpit Jariwala, ${ }^{2}$ and Carlos A. Wigderowitz ${ }^{2}$ \\ ${ }^{1}$ University Department of Orthopaedics and Trauma Surgery, TORT Centre, Ninewells Hospital, Dundee DD1 9SY, UK \\ ${ }^{2}$ NHS Tayside, Dundee DD1 9SY, UK \\ Correspondence should be addressed to Manish Kiran; drmanishkiran@gmail.com
}

Received 14 September 2013; Revised 31 January 2014; Accepted 14 February 2014; Published 17 March 2014

Academic Editor: Padhraig O'Loughlin

Copyright (c) 2014 Manish Kiran et al. This is an open access article distributed under the Creative Commons Attribution License, which permits unrestricted use, distribution, and reproduction in any medium, provided the original work is properly cited.

\begin{abstract}
Background. The aim of this study is to objectively evaluate the quality of cementation by a novel method called the cementation index and assess its utility as a predictor of failure. Materials and Methods. Fifty elbows with primary Coonrad-Morrey total elbow replacement were included. The quality of cementing was assessed by novel methods, the vertical and horizontal cementation index, which were statistically evaluated as predictors of failure. The mean period of followup was $8.08 \pm 2.95$ years (range: from 5.08 to 10.25 years). Results. The mean vertical cementation index of the humerus (vCIH) was $1.22 \pm 0.28$ and that of the ulna (vCIU) was $1.10 \pm 0.18$. Radiolucent zones were noted in two cases in the humerus with a horizontal cementation index of 0.21 and 0.14 , respectively. Both of the cementation indices were not found to be statistically significant predictors of failure $(P>0.05)$. The five-year survival rate was $94 \%$. Discussion and Conclusion. The cementation index, being a ratio, reduces the confounding effect of taking radiographs in different positions of the limb with different magnification in followup radiographs. It is an easy and objective method of assessment of cementation, the results of which need to be validated by a larger study.
\end{abstract}

\section{Introduction}

Total elbow replacement is used in the reconstruction of elbows afflicted by rheumatoid arthritis, osteoarthritis, and trauma. There has been a significant improvement in outcome with changes in the implant design, from the initial linked and fully constrained prostheses to the present-day unlinked and linked, semiconstrained implants. Linked prostheses, like the Coonrad-Morrey implant, have been reported to have better long-term functional outcomes than unlinked implants, like the Souter-Strathclyde prosthesis $[1,2]$. Aseptic loosening is one of the most common causes of failure of the prosthesis in the long term $[3,4]$. Inadequate cementing has been established to contribute to this complication $[5,6]$. The aim of this study is to objectively evaluate cementation by a novel method called the cementation index and assess its utility as a predictor of failure.

\section{Materials and Methods}

Fifty elbows (forty-seven patients), wherein primary total elbow replacement was performed, were included in the study. The Coonrad-Morrey type III prosthesis was used in all cases. The mean age of the patients was sixty-seven years (range from thirty-two to eighty-eight years). The male: female ratio was $20: 30$ elbows. The procedure was performed on the dominant limb in thirty-five elbows and on the righthand side in thirty-one elbows. The data was retrospectively obtained from clinical case record and radiographs. The diagnoses and indications of surgery are shown in Table 1. The postoperative radiographs were evaluated for the vertical cementation index, calculated by dividing the length of the cement mantle by the length of the humeral and ulnar components separately, and the horizontal cementation index, which was calculated by dividing the length of the radiolucent zone greater than $1 \mathrm{~mm}$, if present, seen at the cement-bone interface, by the total length of the cement-bone interface. The vertical cementation index is shown in Figure 1. The followup data included condition of the surgical wound, infection, neurological deficit, range of movement, stability of the prosthesis in axial compression and varus/valgus stress, severity of pain, shift of the implant tip, and failure of the prosthesis. Multiple logistic regression analysis of age, gender, 


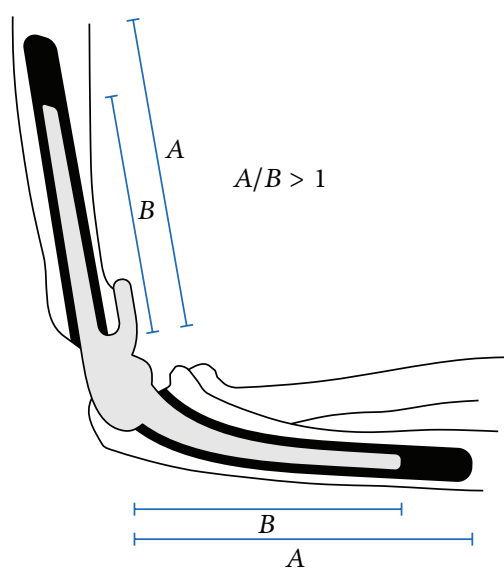

(a)

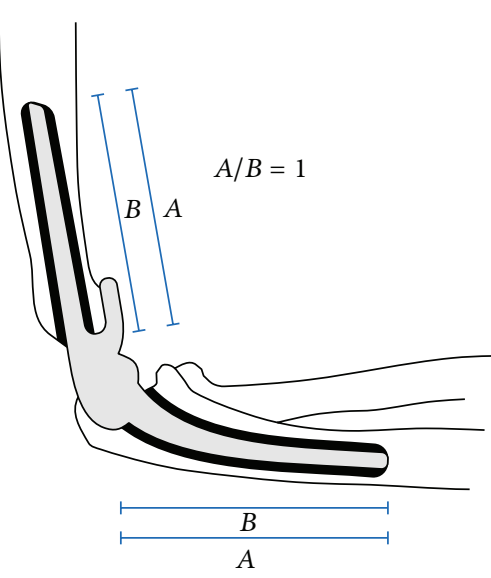

(b)

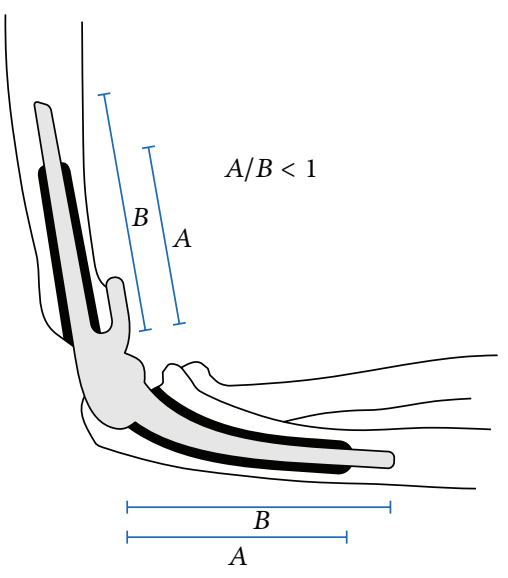

(c)

FIGURE 1: Vertical cementation index.

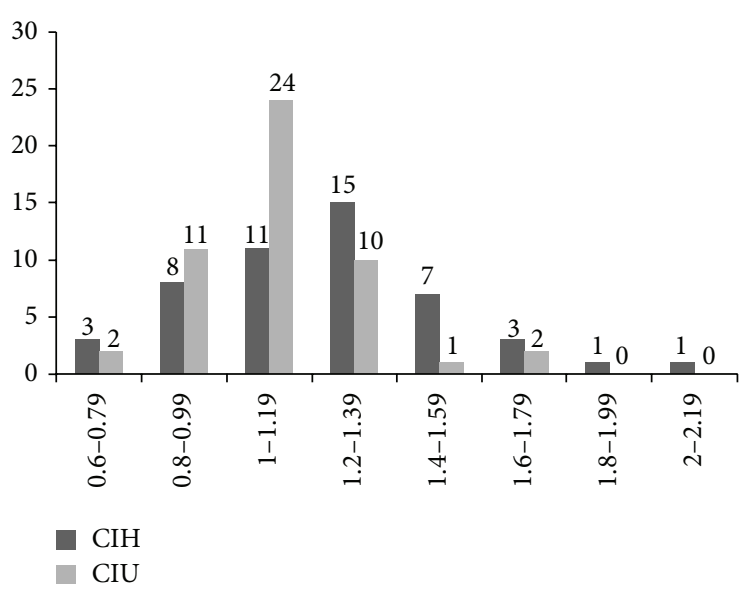

Figure 2: Distribution of vertical cementation index.

diagnosis, side operated upon, limb dominance, and cementation index as predictors of failure was done. The functional and radiological outcome was classified according to Morrey's classification [5]. The mean period of followup was $5.08 \pm 2.95$ years (range from 2.08 to 10.25 years). The mean operative time was $119.08 \pm 18.46$ minutes (range from 70 to 186 minutes).

\section{Results}

Failure of the implant was seen in four cases. The reasons for failure are shown in Table 2. Multiple logistic regression analysis showed that age, gender, side operated upon, dominance of limb, and diagnosis were not significant predictors of failure $(P>0.05)$. The mean vertical cementation index of the humerus ( $\mathrm{vCIH}$ ) was $1.22 \pm 0.28$ (range from 0.7 to 2.1 ) and that of the ulna (vCIU) was $1.10 \pm 0.18$ (range from 0.77 to 1.72 ). The distribution of the two indices is shown in Figure 2. Radiolucent zones were noted in two cases in the humerus with a horizontal cementation index of 0.21 and 0.14 , respectively. Both the vertical and horizontal cementation indices were not found to be statistically significant predictors
TABle 1: Diagnoses and indications for surgery.

\begin{tabular}{lc}
\hline Diagnosis $^{*}$ & Indication for surgery* $^{*}$ \\
\hline Rheumatoid arthritis-29 (58) & Pain-36 (72) \\
Osteoarthritis-5 (10) & Instability-5 (10) \\
Haemophilia-2 (4) & Comminution-8 (16) \\
Acute myeloid leukemia-2 (4) & Tumor-1 (2) \\
Plasmacytoma of distal humerus-1 (2) & \\
Trauma-11 (22) & \\
${ }^{*}$ The values are the number of elbows with the percentage in parenthesis.
\end{tabular}

of failure $(P>0.05)$. The implant that failed due to aseptic loosening has a vertical cementation index of 1.8 and a horizontal cementation index of 0.21 . The five-year survival rate was $94 \%$. The final results were good in thirty-six elbows, fair in eight elbows, and poor in five elbows.

\section{Discussion}

The Coonrad-Morrey prosthesis is an inherently stable linked stemmed implant, due to its toggle-hinge mechanism of 
TABLE 2: Reason for failure.

\begin{tabular}{lcc}
\hline Reason for failure & Number of elbows & Survival in years \\
\hline Aseptic loosening & 1 & 7.4 \\
Deep infection & 2 & 0.583 \\
$\begin{array}{l}\text { Periprosthetic fracture of } \\
\text { the humerus }\end{array}$ & 1 & 0.666 \\
\end{tabular}

articulation. Its design has had significant changes in form, articulation, and coating since its introduction in the 1970s. The first design had a high rate of aseptic loosening which was attributed by the designers of the implant and in subsequent series by other authors to inadequate cementation $[5,6]$. They put forth the opinion that the constraint in the articulating mechanism and the posterosuperiorly directed vector forces acting on the elbow contributed to loosening $[5,7]$. The relatively larger size of the ulnar component in comparison with the ulnar medullary cavity has been postulated to result in inadequate cementation in the ulna [8]. The incidence of aseptic loosening has reduced significantly since the introduction of the anterior flange in the humeral component which can accommodate a bone graft, with some series reporting $100 \%$ five-year survivorship of the implant [2]. There have also been changes in the type of titanium coating of the implant and the introduction of the pin-in-pin articulation. Corradi et al. [6] reported a 15 -year survivorship of $92.4 \%$. There have been reports of ulnar component loosening in literature due to improper placement of the ulnar component and its impingement on the anterior humeral flange [9]. Morrey et al. [5] believed that inadequate cementation was the cause of aseptic loosening in seven of the eleven cases, which were revised for loosening of the implants. Likewise, Corradi et al. [6] reported one case of inadequate cementation of the ulna, which resulted in loosening and revision, seventeen months after the index procedure. Similarly, Schneeberger et al. [9] also noted inadequate cementation in two humeral components and one ulnar component, resulting in loosening of one humeral and one ulnar component. They opined that the antegrade method of cementing resulted in an inadequate cement mantle and recommended a retrograde method of cementation. Aldridge III et al. [3] noted that the cementation was inadequate in one case and marginal in another. However, this did not result in loosening of the implants. Similarly, Prasad and Dent [2] did not observe any aseptic loosening and reported a $100 \%$ survivorship at five years. In our series, we noted a comparable five-year survivorship of the implant. The functional and radiological outcome which was classified according to Morrey's classification [5] was good in a majority of cases in our series. This classification took into account the presence of pain, range of movement, radiological changes like lysis and change in the position of the implant tip, and removal or revision of implants. A painless elbow with a functionally useful range of movement of $100^{\circ}$ [7] was achieved in most cases.

Morrey et al. [5] classified the adequacy of cementation as adequate, marginal, and inadequate based on whether the cement mantle crossed the tip of the prosthesis and the width of the radiolucent zone at bone-cement interface. According to this classification, a case with $10 \%$ coverage of the implant and one with $90 \%$ coverage would both be classified as inadequate cementation, since both of the mantles would not have crossed the tip of the prosthesis. However, one would expect that the clinical outcome of $10 \%$ cement coverage would be different from $90 \%$ coverage. The cementation index is a novel and objective method of assessment of the coverage of the implant with cement, which circumvents this limitation by providing a numerical value to the degree of cementation of the implant. Since it is a ratio, the effect of taking radiographs in different positions of the limb with different magnification in followup radiographs is reduced, thus ensuring comparability. It is very easy to perform, especially with the new computer based imaging systems. The horizontal component of the index has been validated for assessment of the quality of cementing in the femur [10]. It was not found to be a statistically significant predictor of failure in the present study. This result may not be representative, considering the relatively small number of elbows included in the study. Unlike hip and knee replacements, where a larger number of cases are operated upon, most series of total elbow replacement have between forty to seventy cases. A larger study involving multiple centres would provide adequate number of cases to validate the utility of the cementation index as a predictor of failure.

\section{Conclusion}

Cementing techniques contribute to the stability and the long-term outcome of the total elbow prostheses. The cementation index is an easy, reproducible, and objective method of assessment of the adequacy of cementation in stemmed prostheses, the results of which need to be validated by a larger study.

\section{Disclosure}

Caldicott Guardian approval was obtained, University of Dundee.

\section{Conflict of Interests}

None of the authors has any potential or actual personal, financial, political, or competing interest.

\section{References}

[1] K. Güttler, I. Landor, P. Vavř́ík, S. Popelka, A. Sosna, and J. Krásenský, "Total elbow replacement in patients with rheumatoid arthritis," Acta Chirurgiae Orthopaedicae et Traumatologiae Cechoslovaca, vol. 73, no. 5, pp. 423-430, 2011.

[2] N. Prasad and C. Dent, "Outcome of total elbow replacement for rheumatoid arthritis: single surgeon's series with SouterStrathclyde and Coonrad-Morrey prosthesis," Journal of Shoulder and Elbow Surgery, vol. 19, no. 3, pp. 376-383, 2010. 
[3] J. M. Aldridge III, N. R. Lightdale, W. J. Mallon, and R. W. Coonrad, "Total elbow arthroplasty with the Coonrad/CoonradMorrey prosthesis: a 10 to 31-year survival analysis," Journal of Bone and Joint Surgery B, vol. 88, no. 4, pp. 509-514, 2006.

[4] D. R. J. Gill and B. F. Morrey, “The Coonrad-Morrey total elbow arthroplasty in patients who have rheumatoid arthritis: a ten to fifteen-year followup study," Journal of Bone and Joint Surgery A, vol. 80, no. 9, pp. 1327-1335, 1998.

[5] B. F. Morrey, R. S. Bryan, J. H. Dobyns, and R. L. Linscheid, "Total elbow arthroplasty: a five-year experience at the Mayo Clinic," Journal of Bone and Joint Surgery A, vol. 63, no. 7, pp. 1050-1063, 1981.

[6] M. Corradi, M. Frattini, B. Panno, S. Tocco, and F. Pogliacomi, "Linked semi-constrained total elbow prosthesis in chronic arthritis: results of 18 cases," Musculoskeletal Surgery, vol. 94, pp. S11-S23, 2010.

[7] B. F. Morrey, L. J. Askew, K. N. An, and E. Y. Chao, "A biomechanical study of normal functional elbow motion," Journal of Bone and Joint Surgery A, vol. 63, no. 6, pp. 872-877, 1981.

[8] C. P. Little, A. J. Graham, G. Karatzas, D. A. Woods, and A. J. Carr, "Outcomes of total elbow arthroplasty for rheumatoid arthritis: comparative study of three implants," Journal of Bone and Joint Surgery A, vol. 87, no. 11, pp. 2439-2448, 2005.

[9] A. G. Schneeberger, D. C. Meyer, and E. H. Yian, "CoonradMorrey total elbow replacement for primary and revision surgery: a 2 to 7.5-year followup study," Journal of Shoulder and Elbow Surgery, vol. 16, no. 3, pp. S47-S54, 2007.

[10] M. Umar, V. Patil, and S. Lewthwaite, "Validation of a new grading system for femoral cementation in total hip arthroplasty," Journal of Bone and Joint Surgery, vol. 94, no. 37, article 138, 2012. 


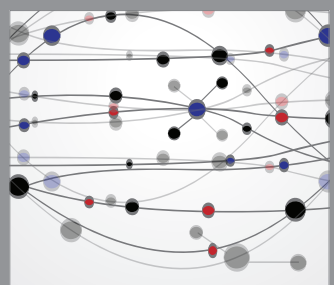

The Scientific World Journal
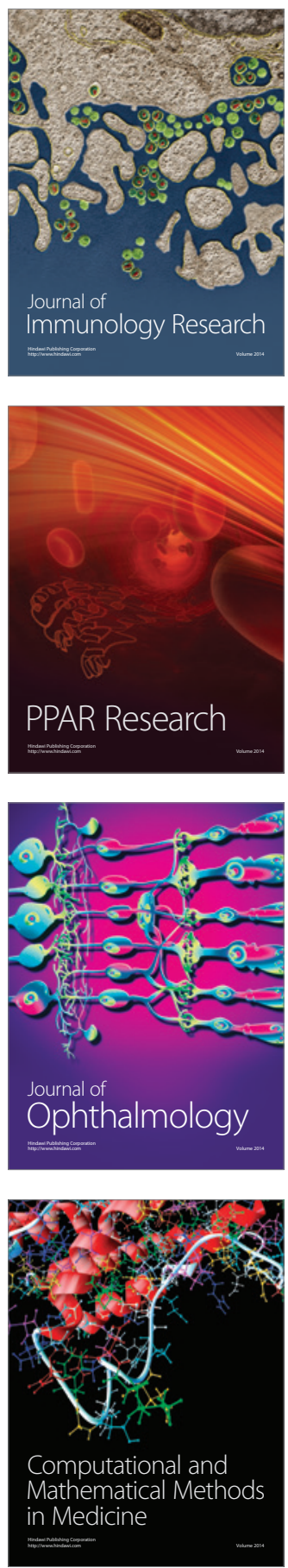

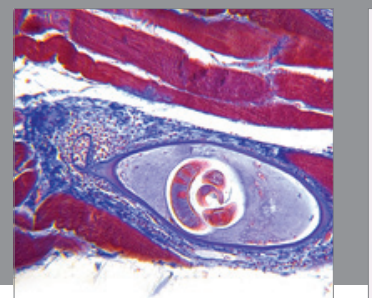

Gastroenterology

Research and Practice
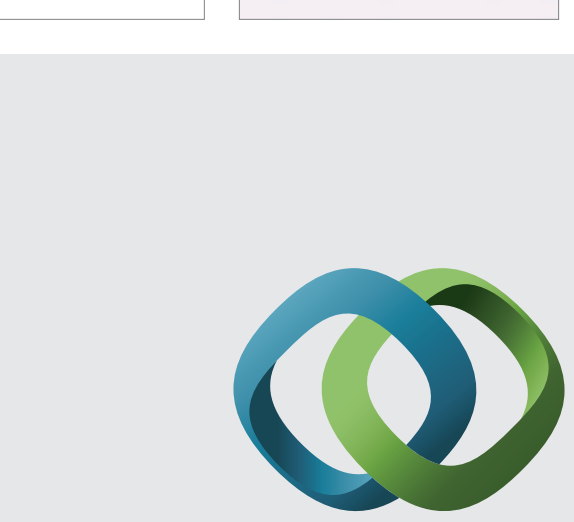

\section{Hindawi}

Submit your manuscripts at

http://www.hindawi.com
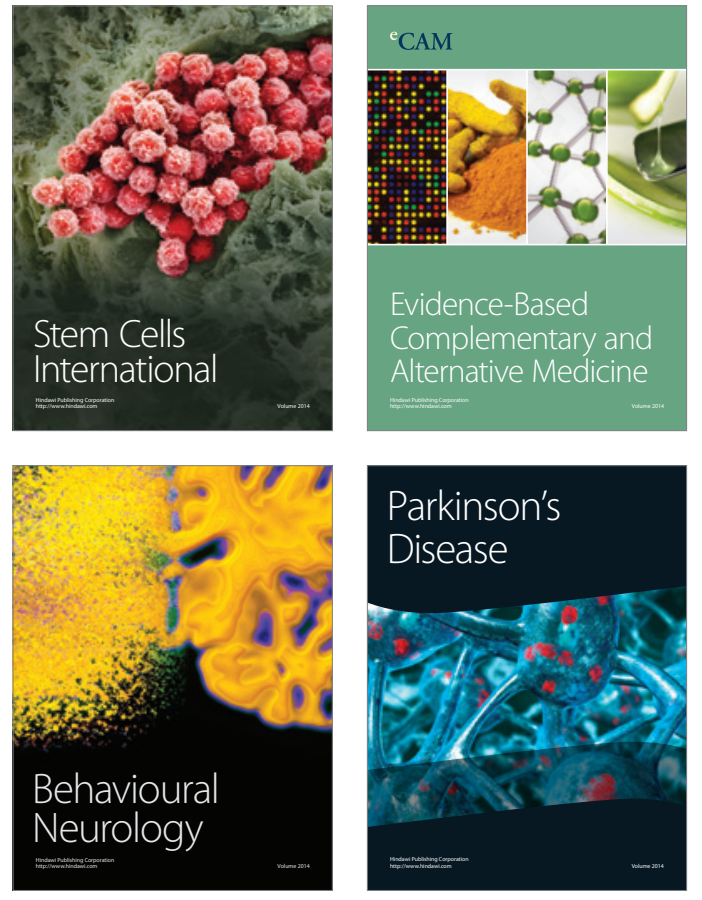
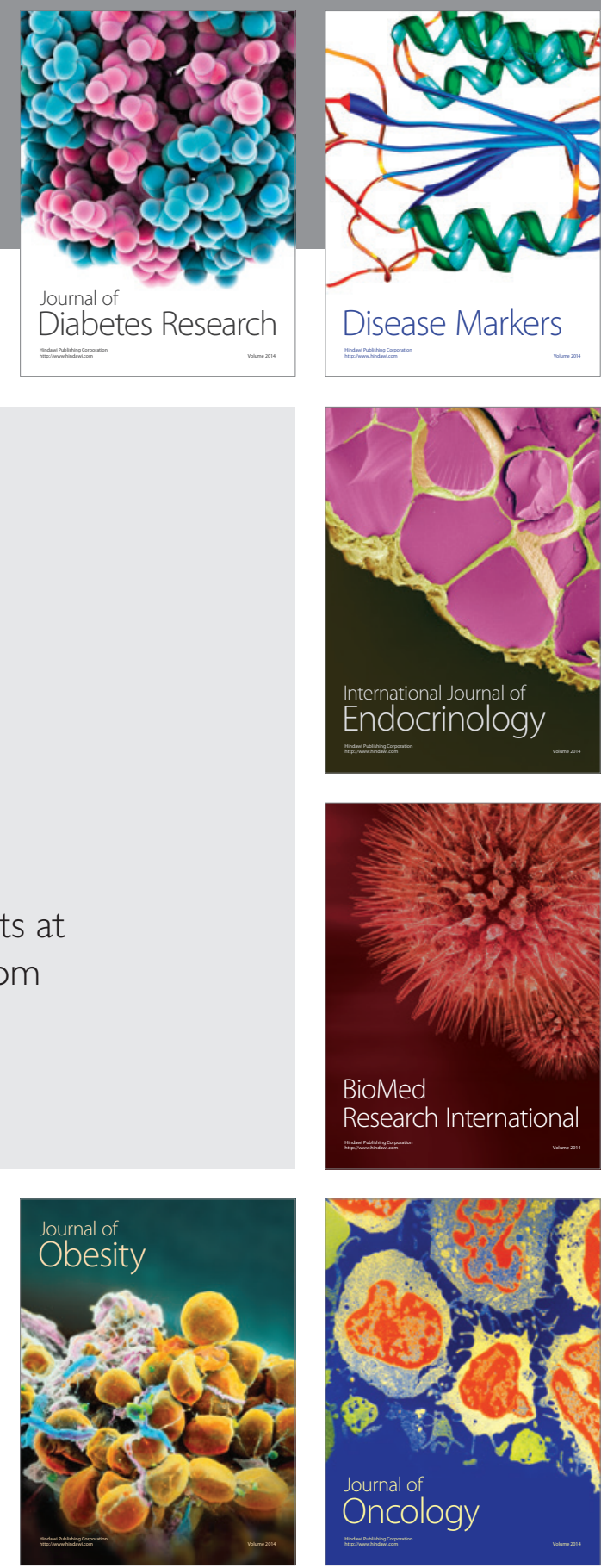

Disease Markers
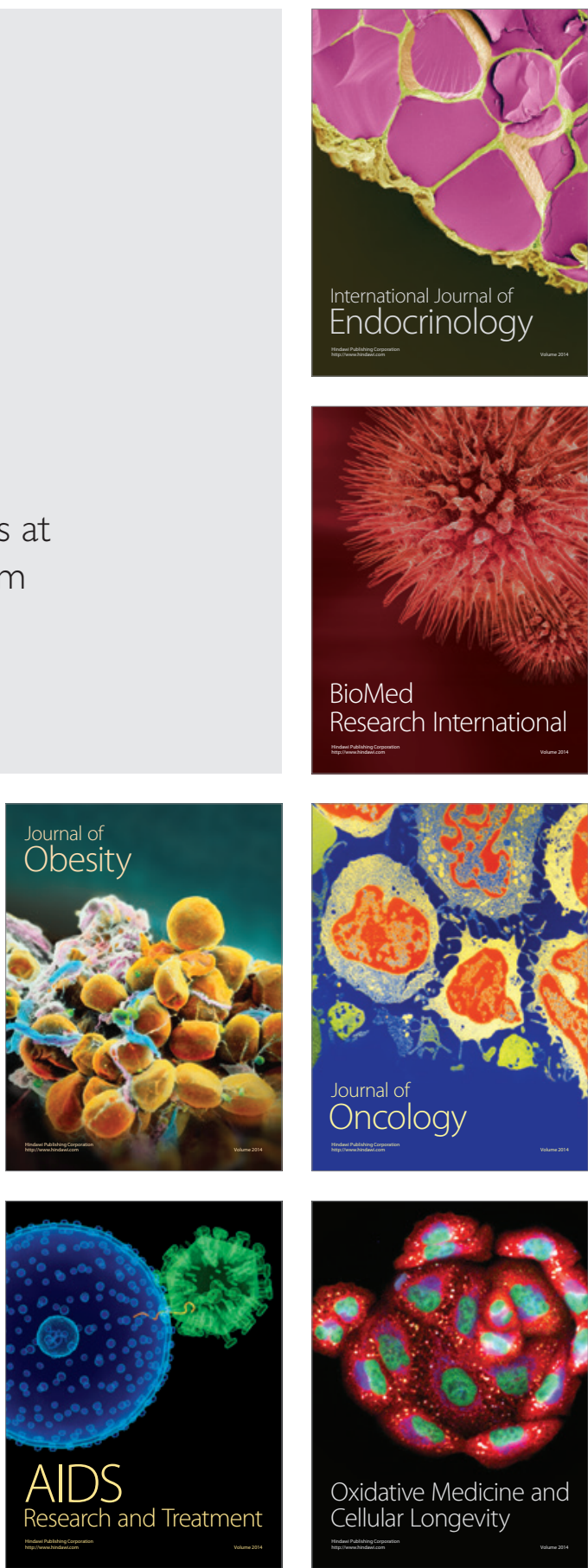